\title{
Avaliação da eficiência da nutrição parenteral quanto à oferta de energia em pacientes oncológicos pediátricos
}

\author{
Evaluation of parenteral nutrition efficiency in \\ supplying energy for pediatric oncology patients
}

Adriana GARÓFOLO'

Susiane Gusi BOIN ${ }^{1}$

Patrícia Claudia MODESTO'

Antonio Sérgio PETRILLI ${ }^{1}$

\section{R E S U M O}

\section{Objetivo}

Avaliar a eficiência da nutrição parenteral administrada a pacientes oncológicos pediátricos quanto à oferta de energia, indicações e principais dificuldades no procedimento.

\section{Métodos}

Estudo observacional descritivo, realizado entre julho de 2003 e julho de 2004 no Instituto de Oncologia Pediátrica da Universidade Federal de São Paulo. Critérios de inclusão: pacientes com câncer recebendo nutrição parenteral nas unidades de internação. A análise da adequação energética foi efetuada usando-se equações preditivas para o cálculo do gasto energético basal ajustado para a progressão inicial.

\section{Resultados}

Foram 41 episódios de nutrição parenteral de, 1.016, internações (4\%): 54\% do sexo masculino, com mediana de uso de 10 dias (1-51). Demonstrou-se que em $56 \%$ dos episódios não se atingiu o gasto energético basal ajustado; $26 \%$ ficaram entre $100 \%$ e $120 \%$ e $18 \%$ acima de $120 \%$. A média do gasto energético basal ajustado foi $106 \%$, com desvio-padrão de $38 \%$ e a mediana $96 \%$. Os motivos associados às dificuldades para atingir o gasto energético basal foram intolerância metabólica, via de nutrição parenteral não exclusiva, suspensão ou interrupção da sua administração, prescrição sem discussão com nutricionista e uso de via periférica. A principal indicação foi por toxicidade gastrintestinal. O volume desprezado de nutrição parenteral demonstrou-se superior a 10\% em 17 de 35 episódios (49\%). Proteção contra luz ambiente (capa), avaliada em 19 pacientes durante os dias da administração, detectou ausência em 37 de 315 dias (12\%).

\footnotetext{
${ }^{1}$ Universidade Federal de São Paulo, Escola Paulista de Medicina, Departamento de Pediatria, Instituto de Oncologia Pediátrica. R. Botucatu, 743, 04023-062, São Paulo, SP, Brasil. Correspondência para/Correspondence to: A. GARÓFOLO. E-mail: <adrigarofolo@hotmail.com>
} 
182 | A. GARÓFOLO et al.

\section{Conclusão}

A oferta de energia não contemplou as necessidades de alta porcentagem de pacientes, principalmente devido à gravidade do quadro clínico, às interrupções e ao uso de via não exclusiva, reduzindo o período disponível para administração da nutrição. A eficiência dos procedimentos por atuação de equipe multidisciplinar poderia reduzir o prejuízo, garantindo uma oferta mais adequada, aumentando os benefícios da nutrição parenteral.

Termos de indexação: neoplasia; gasto energético; nutrição parenteral; terapia nutricional.

\section{A B S T R A C T}

\section{Objective}

The objective of this study was to evaluate parenteral nutrition efficiency in supplying energy for pediatric oncology patients, indications and main difficulties in the procedure.

\section{Methods}

This was an observational descriptive study done from July 2003 to July 2004 at the Pediatric Oncology Institute of the Universidade Federal de São Paulo. Inclusion criteria: hospitalized cancer patients receiving parenteral. Energy adequateness was analyzed by equations that calculated basal energy expenditure adjusted for initial progression.

\section{Results}

Forty-one (4\%) of the 1016 hospitalized patients received parenteral nutrition: 54\% were males and received parenteral nutrition for a median of 10 days (1-51). Fifty-six percent of the episodes did not reach the adjusted basal energy expenditure; 26\% remained between 100 and $120 \%$ and $18 \%$ were above $120 \%$. The mean adjusted basal energy expenditure was $106 \%$ with a standard deviation of $38 \%$ and a median of $96 \%$. The reasons associated with difficulties to achieve the basal energy expenditure were metabolic intolerance, non-exclusive parenteral nutrition, suspension or interruption of its use, prescription without discussing with nutritionist and use of peripheral route. Parenteral nutrition was mainly indicated in gastrointestinal toxicity. The discarded volume of parenteral nutrition was above 10\% in 17 of 35 episodes (49\%). Protection from room light (cover) assessed in 19 patients during the days of administration detected an absence of 37 of 315 days $(12 \%)$.

\section{Conclusion}

Energy supply did not meet the demands of a high percentage of the patients, mainly because of the severity of the clinical scenario, interruptions and non-exclusive use, reducing the amount of time available to administer the nutrition. A multidisciplinary team which would improve the efficiency of the procedures could reduce the losses, guaranteeing a more adequate supply and increasing the benefits of parenteral nutrition.

Indexing terms: neoplasms; energy metabolism; parenteral nutrition; nutrition therapy.

\section{N T R O D U Ç Ã O}

A nutrição parenteral em pacientes com câncer é motivo de grande debate na literatura, devido ao seu uso freqüente, principalmente pelas toxicidades dos medicamentos que desencadeiam efeitos agressivos sobre o trato gastrintestinal ${ }^{1}$.

Assim, a necessidade de nutrir o paciente com câncer que, freqüentemente, apresenta algum grau de desnutrição, associada às dificuldades da alimentação pela via enteral, devido a distúrbios gastrintestinais graves, leva ao seu maior uso. A prática de terapia nutricional em pacientes com câncer visa melhorar a resposta ao tratamento, as chances de cura e a sobrevida sem, no entanto, aumentar os riscos. Porém, a imunossupressão, que ocorre devido ao tratamento antineoplásico, aumenta o risco de infecções associadas à nutrição parenteral ${ }^{2}$.

Apesar da importância da terapia nutricional, o excesso de oferta de energia, que excede a capacidade da sua utilização, aumenta o estresse fisiológico e pode provocar uma sobrecarga metabólica, elevando o gasto energético, que 
ocorre devido ao aumento na produção de catecolaminas. Existe, portanto, uma correlação direta entre oferta de energia por kilograma de peso e aumento na taxa metabólica ${ }^{3,4}$. Prejuízos na função do sistema retículo-endotelial, desconforto respiratório, hipertrigliceridemia súbita, coagulopatia e testes de função hepática anormais também são manifestações da hiperalimentação ${ }^{4}$.

Por outro lado, as repercussões da deficiência de nutrientes são bem reconhecidas. Sabe-se que a hipoalimentação desfavorece o prognóstico, pois, de modo geral, está associada ao maior número de complicações orgânicas e pior resposta ao tratamento. Além disso, pode prejudicar o processo de cicatrização de feridas e a resposta imune, aumentar o risco de infecções nosocomiais, comprometer a função respiratória, prolongar a ventilação mecânica, aumentar as complicações gerais e prolongar o tempo de hospitalização ${ }^{4}$.

Portanto, procedimentos adequados são necessários para reduzir os riscos associados ao uso de nutrição parenteral (NP), bem como otimizar seus efeitos benéficos.

Com o objetivo de avaliar a eficiência do uso da NP administrada em pacientes oncológicos pediátricos quanto à oferta de energia, indicações e principais dificuldades encontradas na sua administração, desenvolveu-se este estudo.

\section{MÉTODOS}

A pesquisa foi desenvolvida no Instituto de Oncologia Pediátrica (IOP) da Universidade Federal de São Paulo (Unifesp), no período de julho de 2003 a julho de 2004, com pacientes hospitalizados para receber tratamento oncológico ou tratar alguma complicação decorrente deste. Tratou-se de um estudo observacional descritivo, que incluiu todos os pacientes com câncer que necessitaram de hospitalização nesse período nas unidades de cuidados gerais/enfermarias (UCG), cuidados intensivos (UCI) e transplante de medula óssea (TMO), e receberam NP.
A coleta de dados referentes à NP foi realizada diariamente pelo nutricionista, por meio de uma ficha clínica com informações referentes a características gerais (idade, sexo e diagnóstico do câncer) e específicas como via de acesso, exclusividade da via, quantidade de glicose, lipídios, aminoácidos e micronutrientes (polivitamínico, oligoelementos e eletrólitos), volume total prescrito, volume total infundido, uso de capa e motivo da indicação da NP. Diariamente também eram descritas as dificuldades encontradas para efetuar a evolução nutricional programada, de acordo com o gasto energético calculado.

A análise dos dados foi efetuada de acordo com a quantidade de energia, oferecida por meio do cálculo da adequação do gasto energético basal (GEB) e do cálculo do GEB ajustado para a progressão inicial, considerando uma evolução de $25 \%, 50 \%$ e $75 \%$ nos três primeiros dias de NP e $100 \%$ nos demais dias. A oferta máxima alcançada no período também foi avaliada (dia de maior oferta de energia administrada). As equações utilizadas foram da Organização Mundial da Saúde (OMS) para crianças e adolescentes até 15 anos e de Harris Benedict (HB) para pacientes com idade superior. O cálculo não incluiu soro glicosado infundido com medicamentos ${ }^{5,6}$.

Também foram coletados dados referentes às indicações do uso de NP, aos motivos associados com as dificuldades em atingir as necessidades de acordo com o cálculo do GEB ajustado, ao volume desprezado e ao uso de capa de proteção contra luz ambiente.

As avaliações da intolerância no metabolismo de glicose e lipídeos, bem como das funções renal e hepática, foram efetuadas por meio de coletas pré-estabelecidas: três vezes por semana e duas vezes por semana, respectivamente.

Tratou-se de um levantamento de dados para um estudo descritivo, cujas variáveis coletadas fazem parte da rotina do acompanhamento de pacientes submetidos à NP. Qualquer avaliação ou procedimento realizado nestes obteve o consentimento dos mesmos e de seus pais ou responsáveis. 
Médias e medianas foram utilizadas para descrever os dados sobre o GEB. Para comparar a oferta de energia ajustada entre os três grupos (UCI, UCG e TMO), o teste de análise de variância para um fator (one way ANOVA) foi aplicado, utilizando-se o programa estatístico NCSS 2000/PASS $2000^{7}$.

\section{RESULTADOS}

Foram 41 episódios de nutrição parenteral (4\%), dos 1.016 episódios de hospitalização: 12 (29\%) na Unidade de Cuidados Intensivos (UCI), 15 (37\%) na Unidade de Transplante de Medula Óssea (TMO) (8 autólogos e 7 alogênicos) e 14 (34\%) na Unidade de Cuidados Gerais/Enfermarias (UCG). Um dos pacientes utilizou NP em duas internações diferentes (UCl e TMO), tendo sido considerado como dois episódios distintos. A distribuição percentual de acordo com o diagnóstico da neoplasia está representada na Tabela 1.

A idade dos pacientes com episódios de NP variou de 2 meses a 18 anos, sendo 54\%

Tabela 1. Distribuição dos pacientes de acordo com os diagnósticos das neoplasias no Instituto de Oncologia Pediátrica, entre julho de 2003 e julho de 2004 (n=41).

\begin{tabular}{lcr}
\hline Diagnóstico & Pacientes (episódios) (n) & $\%$ \\
\hline Leucemia linfoide aguda & 8 & 19,5 \\
Leucemia mielóide aguda & 2 & 5,0 \\
Leucemia bifenotípica & 2 & 5,0 \\
Leucemia mielóide crônica & 1 & 2,4 \\
LMMC juvenil* & 2 & 5,0 \\
Linfoma não-Hodgkin & 5 & 12,2 \\
Linfoma Hodgkin & 1 & 2,4 \\
Tumor de células germinativas & 5 & 12,2 \\
Rabdomiossarcoma & 2 & 5,0 \\
Tumor de Wilms & 1 & 2,4 \\
Sarcoma de Ewing & 1 & 2,4 \\
Neuroblastoma & 1 & 2,4 \\
Tumores de SNC & 4 & 9,8 \\
Outros & 6 & 14,6 \\
\hline Total & 41 & 100,0 \\
\hline
\end{tabular}

LMMC juvenil: leucemia mielomonocícica crônica juvenil; SNC: sistema nervoso central; * Representa dois episódios com o mesmo paciente.
(22/41) do sexo masculino. Dois pacientes (UCI) evoluíram para morte em vigência da NP, assim, dos 41, somente de 39 casos foi possível analisar gasto energético, tempo de uso e dificuldades associadas. A mediana de uso de NP dos 39 pacientes foi de 10 dias (1-51 dias).

As principais indicações de NP em 41 pacientes foram toxicidades gastrintestinais graves, decorrentes do tratamento com quimioterapia e/ou radioterapia (mucosite, esofagite, diarréia, enterite e vômitos) ( $n=23 ; 56,1 \%$ ), intolerância à progressão do volume enteral $(n=5$; $12,2 \%$ ), desnutrição grave com recusa em utilizar sonda enteral $(n=1 ; 2,4 \%)$ e necessidade de jejum prolongado $(n=10 ; 24 \%)$ por: hemorragia ou sangramento digestivo $(n=3)$, íleo paralítico grave $(n=1)$, fístula entérica $(n=1)$, enterectomia por brida em íleo terminal $(n=2)$, risco de perfuração de alça intestinal $(n=1)$, obstrução da cavidade nasal por tumor de rinofaringe $(n=1)$ e pós-cirúrgico de gastroduodenopancreatectomia $(n=1)$. Cinco pacientes estavam em uso de sonda nasoenteral e necessitaram de NP por apresentarem dificuldades em tolerar a progressão do volume da dieta: três devido ao grande volume tumoral em região abdominal e dois por intolerância gastrintestinal devido à quimioterapia.

A avaliação da oferta de energia (não ajustada) na nutrição parenteral dos 39 pacientes durante o período demonstrou que, em média, os pacientes alcançaram 81,1\% (desvio-padrão - DP - de $20,0 \%$ ) do GEB, sendo que $32(82,0 \%)$ destes não atingiram o GEB. A mediana dessa mesma avaliação foi de $76,0 \%$.

A oferta ajustada para os três dias iniciais apresentou média de 106\% (DP=38\%) e, por essa análise, dos 39 pacientes 22 (56\%) não atingiram o GEB ajustado, 10 (26\%) receberam entre 100\% e $120 \%$ e $7(18 \%)$ receberam acima de $120 \%$; 3 (8\%) entre $120 \%$ e $150 \%$ e $4(10 \%)$ acima de $150 \%$ do GEB. A mediana foi de $96 \%$.

A mediana do GEB ajustado foi $90,86 \%$ $(53,7-211,3)$ no grupo $\mathrm{UCl}, 92,20 \%(48-235,7)$ no grupo UCG e 101,70\% (67-145,6) no TMO. Respectivamente, $64,30 \%$ (9/14), 60,00\% (6/10), e 46,70\% (7/15) não atingiram o GEB ajustado. 
A análise que comparou os três grupos (UCI, UCG e TMO) quanto aos valores do GEB ajustado não demonstrou diferença estatística significante. A Figura 1 apresenta o gráfico comparando os três grupos.

Uma oferta máxima de 95\% ( $\mathrm{DP}=27 \%)$ do GEB foi alcançada, em média, para os 39 pacientes com mediana de $93 \%$. Vinte e quatro destes (62\%) não atingiram o GEB, 9 (23\%) receberam entre $100 \%$ e $120 \%, 6(15 \%)$ acima de $120 \%$; e 1 (3\%) acima de 150\%.

Os principais motivos associados às dificuldades para atingir as necessidades energéticas foram intolerância no metabolismo de lipídeos e glicose e alterações hepáticas, via de NP não exclusiva, NP interrompida ou suspensa por procedimentos cirúrgicos, exames ou instabilidade hemodinâmica grave, prescrição de NP sem discussão prévia com o nutricionista e uso de via periférica (Figura 2).

A avaliação do volume desprezado de NP foi realizada em 35 pacientes, demonstrando-se superior a 10\% em 17 (49\%), com média do grupo de $13 \%$.

Proteção contra luz ambiente (capa cobrindo a bolsa de NP) foi avaliada em 19 pacientes durante os dias da sua administração, detectando-se ausência em 37 de 315 dias (12\%).

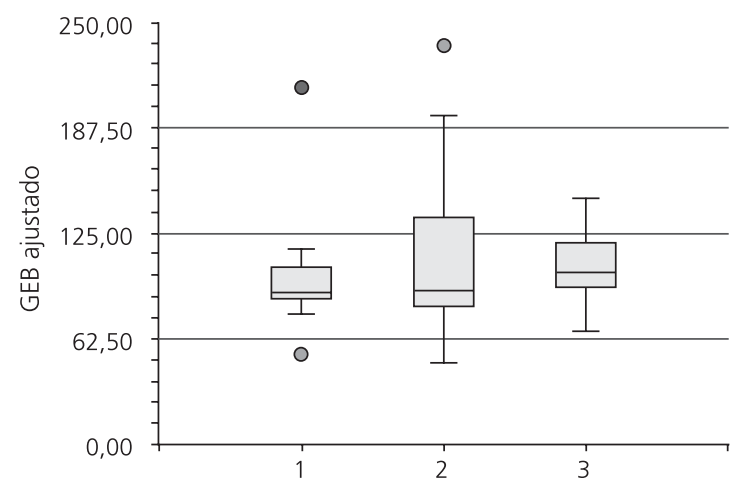

Figura 1. Valores referentes ao gasto energético basal calculado ajustado dos pacientes segundo a unidade de internação no Instituto de Oncologia Pediátrica, São Paulo, junho de 2003 a julho de 2004 (n=39). Dados apresentados como mediana e intervalo de interquartis (Box plot).

1: UCl; 2: UCG/enfermaria; 3: TMO. ANOVA: p>0,05 (não significante).

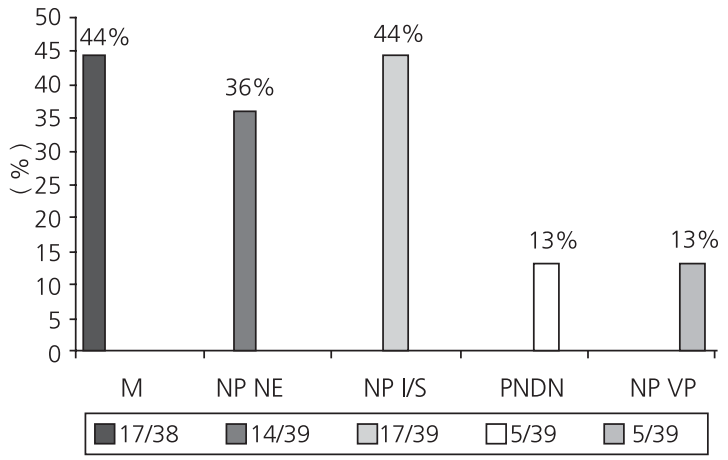

Figura 2. Principais dificuldades avaliadas pela nutricionista durante a coleta dos dados que não permitiram uma evolução da oferta de energia na nutrição parental, impedindo atingir as necessidades energéticas dos pacientes. Instituto de Oncologia Pediátrica, São Paulo, julho de 2003 a julho de 2004 (n=39).

IM: intolerância metabólica (carboidratos e/ou lipídeos); NP NE: nutrição parenteral não exclusiva; NP I/S: nutrição parenteral interrompida ou suspensa; PNDN: prescrição não discutida com o nutricionista; NP VP: nutrição parenteral periférica.

\section{I S C U S S Ã O}

Neste estudo, o uso de nutrição parenteral detectado não se demonstrou elevado, ocorrendo em $4 \%$ dos episódios de internação. Dados sobre o uso de NP em pacientes pediátricos com câncer são escassos. Beghetto et al. ${ }^{8}$ demonstraram 34\% de uso de NP em pacientes hospitalizados em cuidados intensivos, cirurgia e clínica geral, por diferentes diagnósticos. A comparação dos resultados deste estudo com o de Berghetto et al. sugere que o uso da via endovenosa para alimentação foi baixo. Esse resultado poderia estar associado ao maior uso de nutrição enteral precoce, devido à atuação da equipe de nutrição no hospital. De acordo com os resultados do estudo de Gurgueira et al. ${ }^{9}$, a implementação da equipe de terapia nutricional, com importante atuação e constante processo de educação dos profissionais, pode garantir indicações mais precisas de NP, aumentando a terapia por meio da nutrição enteral. No entanto, não é possível descartar que o uso de NP tenha sido aquém do recomendado, pois esse aspecto não foi avaliado. 
Neste estudo, a maioria das indicações de NP esteve associada à toxicidade medicamentosa e a complicações gastrintestinais de grau grave, que prejudicaram ou impossibilitaram o uso da via entérica para alimentação. Em pacientes com câncer, as indicações de NP, geralmente, estão mais associadas com a ocorrência de toxicidade dos medicamentos antineoplásicos sobre o trato gastrintestinal10, demonstrando concordância com os dados desta pesquisa.

Outro aspecto que vale destacar é que pacientes com câncer podem apresentar uma predisposição maior à intolerância da alimentação enteral, devido à redução da motilidade gastrintestinal, ao esvaziamento gástrico e à destruição da mucosa intestinal com redução da capacidade digestiva e absortiva, também associadas ao tratamento antineoplásico. Tais efeitos podem estar exacerbados nos pacientes que necessitam de cuidados intensivos. Treze por cento dos pacientes que usaram NP e nutrição enteral por sonda (NES) foram intolerantes à progressão do volume enteral, provavelmente, pela gravidade da situação clínica.

As necessidades de energia e nutrientes para crianças e adolescentes variam em função da faixa etária. Na criança e no adolescente, a energia é direcionada para a manutenção das necessidades metabólicas e para o crescimento e desenvolvimento. As necessidades metabólicas incluem taxa de metabolismo basal (TMB) e perda de calor para o meio ambiente. Em relação ao adulto, as necessidades na infância e adolescência são três a quatro vezes maiores, sendo que uma parcela substancial da energia diária ingerida (30\% a 40\%) é necessária para o crescimento nos primeiros seis meses de vida, declinando para $2 \%$ a $5 \%$ aos dois anos. Isso significa dizer que a TMB é de, aproximadamente, 50 a $55 \mathrm{kcal} / \mathrm{kg}$ na infância e gradualmente declina para 20 a $25 \mathrm{kcal} / \mathrm{kg}$ por dia durante a adolescência ${ }^{11}$.

Pacientes criticamente doentes, freqüentemente, desenvolvem uma condição aguda de estresse, caracterizada por uma série de alterações metabólicas em resposta a uma variedade de estímulos locais ou sistêmicos da doença. Alguns dos eventos mais importantes associados com essa resposta são resistência à insulina e ao hormônio de crescimento e aumento nas concentrações dos hormônios contra-reguladores, como catecolaminas, glucagon e cortisol. Esses fatores são responsáveis pelo hipercatabolismo e hipermetabolismo observados nessa situação ${ }^{11,12}$.

Embora crianças com doenças graves em cuidados intensivos possam apresentar aumento do gasto energético devido ao estresse metabólico, não utilizam energia para o crescimento e a atividade física. Assim, o cálculo do gasto energético deve considerar a taxa metabólica basal, respeitando as limitações metabólicas do estresse agudo, e contemplar um fator de acréscimo, somente para compensar a agressão ${ }^{11}$.

As equações para o cálculo do GEB em crianças e adolescentes têm demonstrado tanto subestimar como superestimar as necessidades basais de energia, quando comparadas à calorimetria ${ }^{13}$. No estudo de Rodriguez et al..$^{14}$ as equações de Schoefield-HW e da OMS apresentaram maior proximidade com o gasto energético estimado pela calorimetria indireta nessa população.

Entretanto, estudos com pacientes críticos, tanto pediátricos como adultos, têm revelado que as equações não são capazes de estimar o valor do gasto energético igualmente à calorimetria indireta ${ }^{15}$. Em pacientes TMO as equações também têm apontado imprecisões, ora subestimando, ora superestimando as necessidades de energia desses pacientes ${ }^{16-18}$. As equações são utilizadas, portanto, quando a calorimetria não está disponível.

Um acréscimo sobre o GEB, considerando fatores como termogênese, estresse e atividade, é normalmente utilizado. Porém, estudos de gasto energético em pacientes críticos, realizados por calorimetria indireta, verificaram que indivíduos sob dietas normocalóricas apresentam gasto energético total normal ou levemente aumentado, não sendo superior a $20 \%$ do valor basal, calculado 
pela equação de HB. Portanto, o fator recomendado (fator estresse) é 1,2 (GEB 1,2) 1 .

Assim, neste estudo optou-se por utilizar o GEB como medida para avaliar a oferta de energia administrada para os pacientes com câncer.

Nos últimos anos, um excesso na administração de energia e nutrientes tem sido mais freqüentemente observado do que uma oferta insuficiente em pacientes criticamente doentes ${ }^{20}$. Essas complicações demonstram elevar o risco, além de aumentar o custo do tratamento de pacientes graves em, aproximadamente, US $\$ 5,19$ por paciente/dia, justificando a necessidade de melhoria dos métodos para avaliar o gasto energético, reduzindo o desperdício ${ }^{3,4}$. Os efeitos prejudiciais da hiperalimentação resultam, principalmente, em complicações metabólicas, aumento do gasto energético, comprometimento respiratório, disfunção hepática, supressão imunológica e aumento da morbimortalidade ${ }^{21}$.

A sobrecarga de energia, principalmente carboidratos, parece associar-se com o risco de mortalidade. Esse efeito foi avaliado no pós-operatório de adultos, agrupados retrospectivamente com base nos valores de quociente respiratório: >0,95 (grupo hipercalórico: 150\% do gasto energético) versus $<0,95$ (grupo hipoenergético: 100\% do gasto energético). O número de pacientes que desenvolveu sepse ( $14 \%$ versus $4 \%$ ) e a taxa de mortalidade ( $28 \%$ versus $10 \%$ ) foram significantemente maiores no grupo hiperalimentado versus o grupo que recebeu quantidade de energia igual ao gasto energético ${ }^{22}$. Esses dados confirmam a importância da oferta adequada de energia.

Nas prescrições de NP para os pacientes deste estudo observou-se que o excesso na oferta de energia ocorreu em uma porcentagem pequena dos casos. Detectou-se que, apesar de a média da oferta ter atingido $106 \%$ do GEB ajustado, o que significa dizer que a oferta foi o GEB mais um fator estresse de 1,06, aproximadamente, $50 \%$ dos pacientes não receberam quantidade de energia suficiente para alcançar o GEB ajustado e apenas $18 \%$ receberam acima de $120 \%$ do GEB Uma oferta excessiva (hiperalimentação), acima de $150 \%$ do GEB ajustado, foi observada em 10\% das NP. Somente $26 \%$ dos pacientes receberam entre $100 \%$ e $120 \%$ do GEB ajustado, os quais, provavelmente, tiveram oferta mais próxima ao adequado, de acordo com Patiño et al. ${ }^{19}$.

A análise que comparou os três grupos não demonstrou diferenças na porcentagem de energia ofertada, o que pode estar associado ao número pequeno de pacientes do estudo. Seria esperado que pacientes em UCl e TMO apresentassem maior dificuldade na evolução da NP, pela gravidade da condição clínica. Ao contrário, os pacientes em TMO deste estudo tiveram uma oferta energética superior em relação aos demais.

O acompanhamento diário da NP pelo nutricionista, o que é uma rotina do Instituto de Oncologia Pediátrica, poderia justificar os resultados quanto às baixas porcentagens de hiperalimentação neste estudo. Por outro lado, houve uma maior porcentagem de pacientes com oferta aquém do desejado. Algumas razões podem justificar este achado, como as condições clínicas graves dos pacientes, que implicaram em restrição de volume e de oferta de macronutrientes, principalmente carboidratos e lipídeos, devido à intolerância metabólica. Além disso, como descrito nos resultados deste estudo, o uso de via periférica para administração de NP, a via de NP não exclusiva, implicando em interrupções e perdas ou ajustes na taxa de infusão, para correr em período de tempo menor, e a NP interrompida ou suspensa por procedimentos cirúrgicos, exames ou instabilidade hemodinâmica do paciente (esta última confirmando a gravidade da condição clínica), podem ter contribuído para limitar a oferta. Um outro aspecto que vale ressaltar é a realização de algumas prescrições em desacordo com o programado pelo nutricionista (13\%). Apesar de não ter feito parte da avaliação deste estudo, podem-se aventar algumas circunstâncias associadas a esse tipo de ocorrência: falta de conscientização da importância quanto à adequação da oferta de energia, opção em resolver mais rapidamente a 
prescrição, efetuando-se cópia dos valores prescritos no dia anterior e, portanto, atrasando a progressão da oferta, e o excesso de autodidaxia de alguns profissionais. O uso concomitante de nutrição enteral não poderia justificar esses resultados, pois esteve presente em apenas cinco casos, com oferta energética não significativa.

O suporte nutricional em pediatria requer cuidado especial. Num estudo não randomizado, que comparou NP padrão versus NP individualizada, em 46 crianças, observaram-se limitações na oferta nutricional, bem como na possibilidade de realizar alterações na mesma, principalmente quanto a macronutrientes, com o uso da NP padrão ${ }^{23}$. Como comentado anteriormente, a NP não é um método isento de riscos. Portanto, quando há escolha de sua utilização, todos os esforços devem ser efetuados com o intuito de garantir uma oferta nutricional de melhor custo/benefício para compensar o risco.

Assim, as prescrições devem ser realizadas por profissionais especializados, devendo receber respaldo técnico-científico de toda a equipe multidisciplinar. Evidências recentes apontam que a atuação permanente de uma equipe multidisciplinar na prática da NP pode melhorar os resultados clínicos globais ${ }^{9,24,25}$.

Além da detecção da baixa oferta energética descrita, houve uma perda média de 13\% da NP, devido ao volume desprezado. Essa prática implica em riscos de hipoalimentação, principalmente se a equipe não estiver atenta a tal situação. No presente estudo, essa intercorrência esteve associada, provavelmente, com necessidades de pausas por procedimentos ou exames fora da unidade de internação, o que é comum em pacientes com câncer.

Em 12\% dos dias avaliados a ausência de capa de proteção sobre a bolsa de NP foi observada. Essa ocorrência reflete a falta de conscientização e resistência dos profissionais envolvidos nos processos de instalação e administração da NP, já que tal procedimento é rotina do IOP. Por esse motivo, deve-se destacar a importância da atuação efetiva de uma equipe de terapia nutri- cional com profissionais que representam todas as áreas envolvidas diretamente no processo: nutricionistas, farmacêuticos, médicos e enfermeiros. O uso de capas protege o conteúdo da bolsa contra a peroxidação lipídica, evitando perdas nutricionais, bem como a formação de compostos tóxicos para o organismo 26-30.

Se, por um lado, existem os riscos da hiperalimentação, a hipoalimentação também pode prejudicar a recuperação do organismo em estado de hipercatabolismo. A oferta energético-protéica reduzida, por exemplo, pode influenciar negativamente a função imunológica no período de estresse metabólico, reduzir a força dos músculos respiratórios e prejudicar a recuperação de tecidos e órgãos lesados ${ }^{31,32}$. Num estudo recente, Villet et al. ${ }^{33}$ demonstraram que o balanço energético negativo em pacientes de $\mathrm{UCl}$ correlacionou-se com o aumento no número total de complicações e, particularmente, com as complicações infecciosas. O déficit de energia também se correlacionou com a duração da ventilação mecânica, os dias de internação na $\mathrm{UCl}$ e os dias com antibioticoterapia.

Neste estudo, a oferta de energia não contemplou as necessidades, principalmente, devido à intolerância metabólica, provavelmente, pela gravidade do quadro clínico e pausa por instabilidade de alguns pacientes. Entretanto, a falta de padronização dos procedimentos quanto à prescrição, a via de acesso e a administração também prejudicaram a oferta nutricional adequada. Embora o uso de NP não tenha sido alto neste estudo, observou-se que apenas cinco pacientes estavam em uso concomitante de nutrição enteral por sonda, mas sem sucesso.

Apesar de não ter feito parte dos objetivos do estudo, aventa-se a possibilidade de que o uso de NES poderia ter sido otimizado, em alguns casos, por meio de procedimentos padronizados, como o uso de bombas de infusão para dieta, o posicionamento da sonda em porção jejunal, o uso de pró-cinéticos, entre outros cuidados. Por esse aspecto não ter sido avaliado, não se sabe se nos demais casos (que não usaram NES), a 
passagem e/ou o uso da sonda estavam realmente contra-indicados. A melhoria desse procedimento poderia contribuir para que a oferta das necessidades de energia fosse atingida.

Portanto, a eficiência dos procedimentos e a atuação da equipe multidisciplinar, provavelmente, podem garantir uma oferta mais adequada e contribuir para melhorar os resultados, reduzindo os prejuízos e aumentando os benefícios da terapia nutricional.

\section{O N C L U S Ã O}

A oferta de energia não contemplou as necessidades de alta porcentagem de pacientes, principalmente devido à gravidade do quadro clínico, às interrupções e ao uso de via não exclusiva, reduzindo o período disponível para administração da nutrição. A eficiência dos procedimentos, por atuação de equipe multidisciplinar, poderia reduzir o prejuízo, garantindo uma oferta mais adequada e aumentando os benefícios da nutrição parenteral.

\section{REFERÊ N CIAS}

1. Garófolo A. Diretrizes para terapia nutricional em crianças com câncer em situação crítica. Rev Nutr. 2005; 18(4):513-27.

2. Meadows N. Monitoring and complications of parenteral nutrition. Nutrition. 1998; 14(10):806-8.

3. McClave AS, Lowen CC, Kleber MJ, Nicholson JF, Jimmerson SC, McConnell JW, et al. Are patients fed apropriately according to their caloric requirements? JPEN. 1998; 22(6):375-81.

4. McClave SA, McClain CJ, Snider HL. Should indirect calorimetry be used as part of nutritional Assessment? J Clin Gastroenterol. 2001; 33(1): 14-9.

5. Garófolo A. Avaliação nutricional e requerimentos. In: Petrilli AS, Carvalho BW, Lee JH. Cuidados intensivos no paciente oncológico pediátrico. São Paulo: Atheneu; 2003. p.213-30.

6. Children's Oncology Group, Cancer Control: Nutrition Sub-Committee. Algorithm for nutrition intervention and categories of nutritional status in the pediatric oncology patient-references and resources. In Children's Oncology Group (COG) Symposium. 2004; Washington (DC); 2004.

7. Dawson B, Trapp RG. Bioestatística básica e clínica. 3a. ed. Rio de Janeiro. McGraw-Hill; 2003.

8. Beghetto MG, Victorino J, Teixeira L, Azevedo MJ. Parenteral nutrition as a risk factor for central venous catheter-related infection. JPEN. 2005; 29(5):367-73.

9. Gurgueira GL, Leite HP, Taddei JAAC, Carvalho WB. Outcomes in a pediatric intensive care unit before and after the implementation of a nutrition support team. JPEN. 2005; 29(3):176-85

10. American Society for Parenteral and Enteral Nutrition. Board of Directors and The Clinical Guidelines. Safe practices for parenteral nutrition formulations. National Advisory Group Standards and Practice Guidelines for Parenteral Nutrition. Special Report. JPEN. 1998; 22(2):1-41.

11. Chwals WJ. Overfeeding the Critically III Child: Factor Fantasy? New Horizons. 1994; 2(2):147-55.

12. Grimminger F, Seeger W, Mayer K. Use of n-3 fatty acid-containing lipid emulsion in the Intensive Care Unit environment: the clinician's view. Clin Nutr. 2002; 21(S2):23-9.

13. Wong WW, Butte NF, Hergenroeder AC, Hill RB, Stuff JE, Smith EO. Are basal metabolic rate prediction equations appropriate for female children and adolescents? J Appl Physiol. 1996; 81(6):2407-14.

14. Rodriguez G, Moreno LA, Sarria A, Fleta J, Bueno $M$. Resting energy expenditure in children and adolescents: agreement between calorimetry and prediction equations. Clin Nutr. 2002; 21(3):255-60.

15. Coss-Bu JA, Jefferson LS, Walding D, David Y, Smith $E O$, Klish WJ. Resting energy expenditure in children in a pediatric intensive care unit: comparison of Harris-Benedict and Talbot predictions with indirect calorimetry values. Am J Clin Nutr. 1998; 67(1):74-80.

16. Hutchinson M, Clemans GW, Springmeyer SC, Flournoy N. Energy expenditure estimation in recipients of marrow transplants. Cancer. 1984; 54(8):1734-8.

17. Chamouard CV, Chambrier C, Michallet M, et al. Energy expenditure during alloneic and autologous bone marrow transplantation. Clin Nutr. 1998; 17(6):253-7.

18. Ringwald-Smith K, Williams R, Horwitz E, Schmidt $M$. Determination of Energy Expenditure in Bone Marrow Transplant Patient. NPC. 1998; 13:215-8.

19. Patiño JF, Pimiento SE, Vergara A, et al. Hypocaloric support in the critically ill. World J Surg. 1999; 23(6): 553-9.

20. Frankenfield DC, Wiles CE, Bagley S, Siegel JH. Relationships between resting and total energy 
expenditure in injured and septic patients. Crit Care Med. 1994; 22(11):1796-804.

21. Klein CJ, Stanek GS, Wiles CE. Overfeeding macronutrients to critically ill adults: metabolic complications. J Am Diet Assoc. 1998; 98(7): 795-806

22. Vo NM, Waycaster M, Acuff RV, et al. Effects of postoperative carbohydrate overfeeding. Am Surg. 1987; 53(11):632-5.

23. Guenter P, Curtas S, Murphy L, Orr M. The impact of nursing practice on the history and effectiveness of total parenteral nutrition. JPEN. 2004; 28(1): 54-9.

24. Naylor CJ, Griffiths RD, Fernandez RS. Does a multidisciplinary total parenteral team improve patient outcomes? A systematic review. JPEN. 2004; 28(4):251-8

25. Krohn K, Babl J, Reiter K, Koletzko B. Parenteral nutrition with standard solutions in paediatric intensive care patients. Clin Nutr. 2005; 24(2): 274-80.

26. Lavoie JC, Belanger S, Spalinger M, Chessex P. Admixture of a multivitamin preparation to parenteral nutrition: the major contributor to in vitro generation of peroxides. Pediatrics [cited 2003 Out 12]. 1997; 99(3). Available from: http:// www.pediatrics.org/cgi/content/full/99/3/e6

27. Steger PJK, Muhlebach SF. Lipid peroxidation of intravenous lipid emulsion and all-in-one admixtures in total parenteral nutrition bags: the influence of trace elements. JPEN 2000; 24(1):37-41.

28. Allwood MC. Light protection during parenteral nutrition infusion: is it really necessary? Nutrition. 2000; 16(3):234-5.

29. Allwood MC, Martin HJ. The photodegradation of vitamins $A$ and $E$ in parenteral nutrition mixtures during infusion. Clin Nutr. 2000; 19(5):339-42.

30. Chessex P, Lavoie JC, Rouleau T, et al. Photooxidation of parenteral multivitamins induces hepatic steatosis in a neonatal guinea pig model of intravenous nutrition. Pediatr Res. 2002; 52(6): 958-63.

31. Mota EM, Garcia PCR, Piva JP, Fritscher CC. A influência da desnutrição na utilização de ventilação mecânica em crianças admitidas em UTI pediátrica. J Pediatr. 2002; 78(2):146-52.

32. Casey G. Nutritional support in wound healing. Nurs Standard. 2003:17(23):55-8.

33. Villet S, Chiolero RL, Bollmann MD, Revelly JP, Cayeux MC, Delarue J, et al. Negative impact of hypocaloric feeding and energy balance on clinical outcome in ICU patients. Clin Nutr. 2005; 24(4): 502-9.

Recebido em: 20/10/2005

Versão final reapresentada em: 1/9/2006 Aprovado em: 3/10/2006 